\title{
A Rare Case of Submandibular Lymphadenopathy Due To Pediculosis Pubis Infestation of Facial Hair - A Case Report
}

\author{
Krishna Prasanth*, Kalpana Ramachandran*, Jhanshi Charles**, \\ Prakash Dhanavelu", Ganesh Arumugam ${ }^{\$}$ \\ "Department of Epiemiology, The Tamilnadu Dr.MGR Medical University \\ **Registrar, The Tamilnadu Dr.MGR Medical University \\ ${ }^{\text {\# Department of Oral Surgery, Sree Balaji Dental College }}$ \\ ${ }^{\$}$ Department of Experimental Medicine, The Tamilnadu Dr.MGR Medical University
}

\begin{abstract}
Sexually transmitted infections have become a major public health problem in the global scenario. Use of contraceptives such as condoms can prevent exchange of body fluids thereby preventing transmission of certain sexually transmitted infections, however many other sexually transmitted infections gets transmitted through direct body contact. Pediculosis pubis is an uncommon sexually transmitted infection caused by the obligate ectoparasite Phthirus pubisor Crab lice.

This case report of a 21 years old male patient who presented with submandibular lymphadenopathy and multiple erythematous macules on his face, chest and pubic region. He gave a history of multiple sexual exposures. Further examination and investigations revealed it to be a case of Pediculosis pubis. This parasite was collected, examined macroscopically, microscopically, and morphologically identified as Phthirus pubis
\end{abstract}

Key Words: Pediculosis pubis, submandibular lymphadenopathy, erythematous macules

\section{Introduction}

Pediculosis is infestation of lice on the human body. It can occur on any part of the body. The most common types are Pediculosis capitis, Pediculosis corporis and Pediculosis pubis ${ }^{1}$. Pediculosis pubis is caused by infestation of the lice Phthirus pubis. Cases of Pediculosis pubis have been associated with Sexual intercourse with infected partner or by contact with shared towels, bed spreads or clothing. ${ }^{2}$ Phthirus pubis is primarily found in the pubic hair but on rare occasion can spread to axilla, beard, eyebrows, eyelashes or body hair. ${ }^{1}$ There is dearth of knowledge regarding diagnosis of pediculosis pubis infection and most often it is misdiagnosed. ${ }^{3}$ This is a case report of lymphadenopathy of submandibular lymph nodes secondary to pediculosis pubis infection of facial hair.

\section{Case History}

A 21 years old, unmarried male patient presented to the dental clinic with chief complaints of pain and swelling in the neck near the angle of the jaw on left side since 15 days. He also complained of occasional fever, itching on face, chest and pubic region since 5 months. The patient also gave a history of unprotected sex with multiple homosexual partners.

\section{Examination}

On general examination the patient was conscious, well built with normal gait and posture. He was mildly febrile (99.6 F). Extra oral examination revealed lymphadenopathy in the left submandibular region. Lymph nodes were firm and tender on palpation. Examination of the face, axilla and pubic region demonstrated multiple pruritic erythematous macules. Careful examination through magnifying lens showed multiple yellowish- brown colored insects of size 1 to $1.2 \mathrm{~mm}$. The insects were motile and multiple nits were visible in the chest and pubic region. Intra oral examination revealed dental caries in relation to tooth number 16, 17 and 47. Stains and calculus were present. All other findings were normal and non-contributory.

\section{Investigations}

The insects were collected with tweezers for parasitological investigations. Macroscopic and microscopic examination confirmed the insects to be Phthirus pubis. The patient was also advised blood investigations including CBC, ELIZA and VDRL to rule out other sexually transmitted infections like HIV and syphilis. CBC indicated Anemia (10.1\%), Neutrophilia (79\%), Lymphocytosis (53\%) and Eosinophilia (9\%). All other findings were normal and noncontributory. 


\section{Management}

The patient was advised to abstain from unsafe sexual practice with multiple partners and was educated about the parasite and its treatment. Tablet. Ivermectin $12 \mathrm{mg}$ was prescribed orally as a single dose and to be repeated the next week. Permethrin 1\% solution was advised for topical use. Antibiotics Amoxycicillin $500 \mathrm{mg}$ and potassium clavulanate $125 \mathrm{mg}$ t.i.d, analgesics Aceclofenac 100mg and paracetamol 500mg combination b.d for 7 days was prescribed for lymphadenopathy. Patient was also told to disinfect his bed and clothing.

Patient was advised to follow up after a week which revealed completely cured lymphadenopathy and erythematous macules. Two weeks follow up showed complete treatment from itching and lice.

\section{Discussion}

Pthirus pubis infestation in humans is called as Pediculosis pubis. Pthirus pubis is an ectoparasite of Anoplura group and Pediculidae family. This infection can occur at any age; however it is more common in sexually active age group (18-35 yrs). ${ }^{3}$ Gavin hart et al suggested that pediculosis pubis in men is associated with homosexuality and sexual exposure with multiple partners. ${ }^{6}$ In this present case, the patient's history of multiple homosexual exposures is a risk factor for pediculosis pubis.

Pthirus pubis occurs in regions rich in apocrine glands and hence pubic region, axillae, eyebrows, eyelashes, mustache, beard, perianal region etc..., are commonly involved. ${ }^{2}$ It can spread during vaginal, anal or oral sex.In our case, multiple insects were found in beard, chest, axilla and pubic region. Multiple nits were also visible in the pubic region. Pthirus pubis feeds on human blood from cutaneous capillaries and reproduce by laying nits which are adherent to the base of hair shaft. ${ }^{3}$ An adult female insect lay about 3 to 4 nits per day, which hatch in 1 week. ${ }^{10}$ After undergoing several developmental stages, the adult insect is formed in about 2 weeks. ${ }^{10}$ The entire life cycle of the insect is around 30 days. ${ }^{10}$ The injected saliva of the insect causes hypersensitivity reactions in the form of wheals or papules. Major symptom of Pediculosis is pruritus. ${ }^{2,3}$ Excoriations associated with pruritus may lead to secondary infections like pyoderma which may lead to fever and regional lymphadenitis in severe cases. ${ }^{2}$ In this present case multiple erythematous macules were found all over the body and the patient had submandibular lymphadenopathy on the left side. Involvement of the submandibular lymphnodes secondary to pediculosis pubis, previously reported is rare. Moreover the noncontributory dental findings support the diagnosis of submandibular lymphadenopathy secondary to pediculosis pubis.

Pthirus pubis can be seen macroscopically and microscopically. ${ }^{2}$ The insect appear flattened with three pairs of legs, of which the last two pairs are adapted with specialized claws that help them adhere to hair shaft. They measure 0.8 to $1.2 \mathrm{~mm}$ in length and appear yellow to light brown which makes it difficult to spot. ${ }^{1}$ Diagnosis is usually by physical examination which reveals multiple live insects or nits adhering to base of hair shaft. ${ }^{1,2}$ Dermatoscopy may be useful in many cases. ${ }^{2}$ Pediculosis is primarily sexually transmitted and often occurs in multiple partner involved sex; hence screening for other sexually transmitted infection is mandatory. 8,9 According to Hermann Feldmeier\&JorgHeukelbach (2008) Epidermal parasitic skin infections are neglected by health care providers and efforts to control these infectionsat the community level have rarely been undertaken.

Pediculosis is the most contagious sexually transmitted infection with $95 \%$ chance of acquiring the infection after one sexual exposure. ${ }^{5}$ There is dearth of literature regarding the epidemiology of pediculosis pubis infection $^{3}$, particularly in India. Although morbidity associated with pediculosis is high, assessment regarding the burden of disease does not exist. This is the first case report of submandibular lymphadenopathy associated with pediculosis pubis.

In conclusion, this case highlights the importance of parasitological investigation as a part of regular history taking and case diagnosis in dental as well as medical set up. It also emphasizes the importance of health education to the public, especially young adults as these ectoparasitic infections are sexually transmitted and are at a risk for other STIs. Cases like the present scenario may be atypical and often may be misguiding. This requires multidisciplinary approach by dermatologist and dentist for prompt diagnosis and management. 

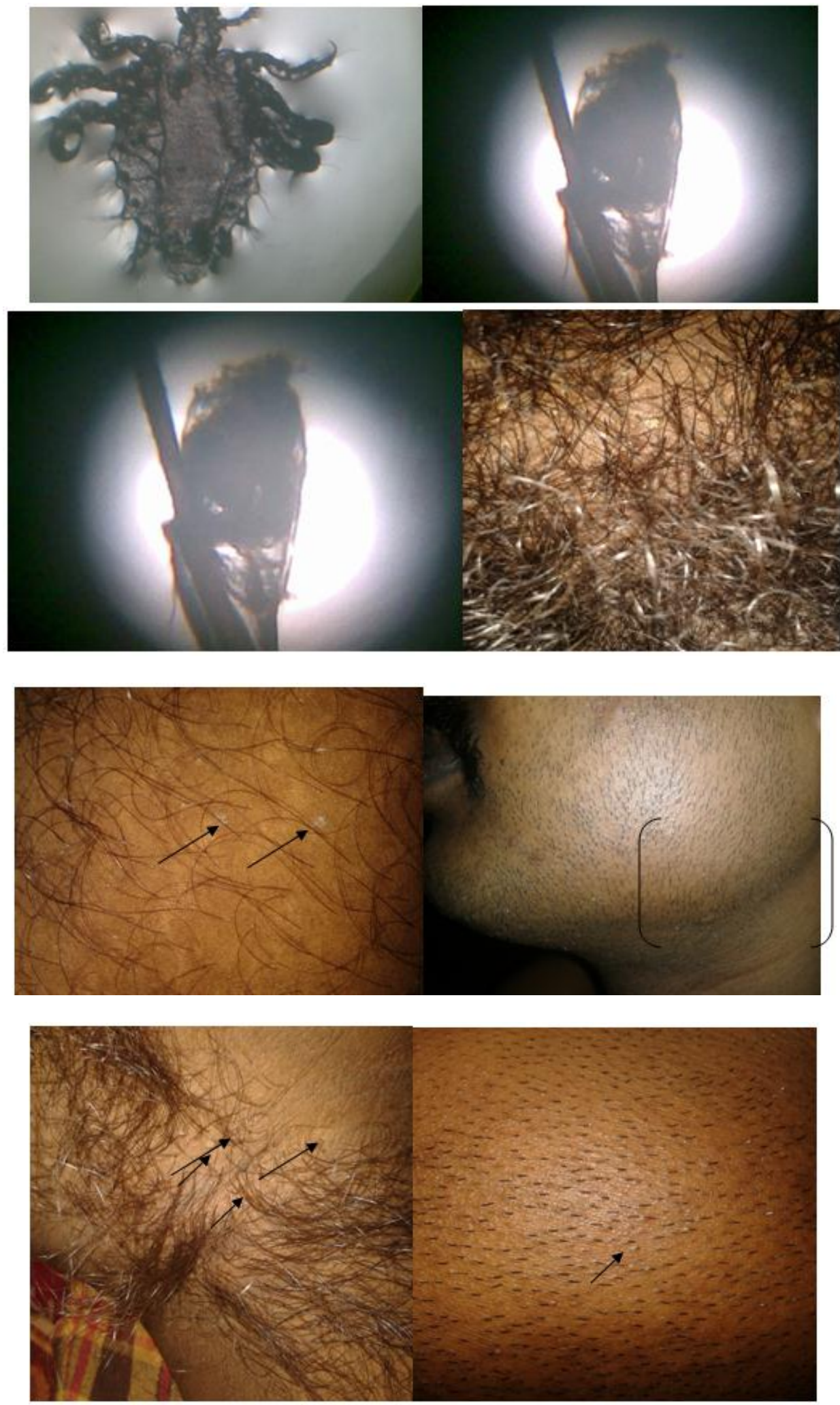

References

[1]. Pediculosis, Christine J. Ko, and Dirk M. Elston, Journal Of American Academy of Dermatology January 2004

[2]. Pediculosis Pubis and Dermoscopy, et al Acta Dermato venerol Croat 2009;17(1):77-83

[3]. The Presence of the Pubic Louse Pthirus Pubis in Two Patients Complaining from Tick Infestation Ali Beytur et al Eur J Gen Med 2011;8(2):160-2

[4]. Pediculosis pubis: presentation to ophthalmologist as pthriasispalpebrarum associated with corneal epithelial keratitis, N.P.Manjunatha et al, International Journal of STD \& AIDS 2006; 17: 424-426

[5]. Infectious diseases of female genital tract, fifth edition by Richard.L. Sweet, Ronald.s. gibbs 
A Rare Case of Submandibular Lymphadenopathy Due To Pediculosis Pubis Infestation of ......

[6]. Factors Associated with pediculosis pubis and scabies-Gavin Hart, Enitourin Med 1992;68:294-295

[7]. Communicable disease management protocol Pediculosis (head, body or crab lice), Communicable disease control unit,Mannetoba public health, November 2001

[8]. Pubic Lice (Pthirus pubis): History, Biology and Treatment vs. Knowledge and Beliefs of US College Students Alice L. Anderson and Elizabeth Chaney, Int. J. Environ. Res. Public Health 2009, 6, 592-600

[9]. Fisher I, Morton RS. Phthirus pubis infestation. Br J Vener Dis 1970;46:325-9

[10]. Nuttall GHF. The biology of Phthirus pubis. Parasitology 1918;10:383-405 